Article

\title{
Empagliflozin Protects HK-2 Cells from High Glucose-Mediated Injuries via a Mitochondrial Mechanism
}

\author{
Wen-Chin Lee ${ }^{1}{ }^{1}$, You-Ying Chau ${ }^{2}$, Hwee-Yeong $\mathrm{Ng}^{1}{ }^{1}$, Chiu-Hua Chen ${ }^{1}$, Pei-Wen Wang ${ }^{3}$, \\ Chia-Wei Liou ${ }^{4}$, Tsu-Kung Lin ${ }^{4}$ and Jin-Bor Chen $1, *(\mathbb{D}$
}

1 Division of Nephrology, Department of Internal Medicine, Kaohsiung Chang Gung Memorial Hospital and Chang Gung University College of Medicine, Kaohsiung 83301, Taiwan; leewenchin@gmail.com (W.-C.L.); stan@cgmh.org.tw (H.-Y.N.); becky0917@cgmh.org.tw (C.-H.C.)

2 Centre for Cardiovascular Science, The Queen's Medical Research Institute, University of Edinburgh, Edinburgh EH16 4TJ, UK; You-Ying.Chau@ed.ac.uk

3 Division of Metabolism, Department of Internal Medicine, Kaohsiung Chang Gung Memorial Hospital and Chang Gung University College of Medicine, Kaohsiung 83301, Taiwan; wangpw@ms18.hinet.net

4 Department of Neurology, Kaohsiung Chang Gung Memorial Hospital and Chang Gung University College of Medicine, Kaohsiung 83301, Taiwan; cwliou@ms22.hinet.net (C.-W.L.); tklin@adm.cgmh.org.tw (T.-K.L.)

* Correspondence: chenjb1019@gmail.com; Tel.: +886-7-731-7123 (ext. 8306)

Received: 9 August 2019; Accepted: 13 September 2019; Published: 14 September 2019 updates

\begin{abstract}
Empagliflozin is known to retard the progression of kidney disease in diabetic patients. However, the underlying mechanism is incompletely understood. High glucose induces oxidative stress in renal tubules, eventually leading to mitochondrial damage. Here, we investigated whether empagliflozin exhibits protective functions in renal tubules via a mitochondrial mechanism. We used human proximal tubular cell (PTC) line HK-2 and employed western blotting, terminal deoxynucleotidyl transferase dUTP nick end labelling assay, fluorescence staining, flow cytometry, and enzyme-linked immunosorbent assay to investigate the impact of high glucose and empagliflozin on cellular apoptosis, mitochondrial morphology, and functions including mitochondrial membrane potential (MMP), reactive oxygen species (ROS) production, and adenosine triphosphate (ATP) generation. We found that PTCs were susceptible to high glucose-induced mitochondrial fragmentation, and empagliflozin ameliorated this effect via the regulation of mitochondrial fission (FIS1 and DRP1) and fusion (MFN1 and MFN2) proteins. Empagliflozin reduced the high glucose-induced cellular apoptosis and improved mitochondrial functions by restoring mitochondrial ROS production, MMP, and ATP generation. Our results suggest that empagliflozin may protect renal PTCs from high glucose-mediated injuries through a mitochondrial mechanism. This could be one of the novel mechanisms explaining the benefits demonstrated in EMPA-REG OUTCOME trial.
\end{abstract}

Keywords: empagliflozin; mitochondria; proximal tubular cell; diabetic kidney disease

\section{Introduction}

Diabetic kidney disease (DKD) affects approximately $40 \%$ of patients with diabetes and has emerged as a leading cause of chronic kidney disease worldwide [1-3]. Empagliflozin, one of the sodium-glucose cotransporter 2 (SGLT2) inhibitors, has recently been reported to retard the progression of DKD in EMPA-REG OUTCOME trial [4]. Animal studies have shown that empagliflozin improves renal functions [5] and is associated with reduced hyperfiltration, albuminuria [6], and ameliorated pathology [7]. However, the mechanisms underlying these effects remain largely unknown. The proposed mechanisms include weight loss, natriuresis, and renal tubuloglomerular feedback [8]. 
The production of reactive oxygen species (ROS) is increased in diabetes [9], resulting in mitochondrial insult and altered mitochondrial genetics and functions. This mitochondriopathy has emerged as a crucial phenomenon in DKD, owing to known changes in the mitochondrial DNA copy number in the leukocytes [10], altered expression of mitochondrial proteins in DKD patient kidneys, and enlarged mitochondria in the renal proximal tubules of animal models and human renal biopsy tissues [11].

Oxidative stress-mediated mitochondrial injuries result in cellular dysfunction and even death via apoptotic pathways. The mitochondrial morphology is shown to be altered in response to these processes. Mitochondrial morphological dynamics are determined by the balance between two opposing processes, namely, fission and fusion. The key proteins involved in mitochondrial fusion include mitofusin 1 (MFN1) and mitofusin 2 (MFN2) [12,13], while dynamin-related protein-1 (DRP1) and mitochondrial fission 1 protein (FIS1) are associated with the mitochondrial fission. High glucose induces mitochondrial fragmentation and cellular apoptosis in renal proximal tubules [14]. As renal proximal tubular cells (PTCs) are the direct site of action for SGLT2 inhibitors, here we used human kidney PTCs to investigate whether SGLT2 inhibitors offer direct renal protection via a mitochondrial mechanism.

\section{Materials and Methods}

\subsection{Cell Culture and Chemicals}

HK-2 cells (ATCC CRL-2190, BCRC 60097, Taiwan) were incubated in culture plates without filters in a humidified atmosphere with $5 \% \mathrm{CO}_{2}$ at $37^{\circ} \mathrm{C}$. The culture medium comprised of $500 \mathrm{~mL}$ of keratinocyte medium (Gibco; Thermo Fisher Scientific, Inc., Waltham, MA, USA), 25 mL fetal bovine serum (FBS; Gibco; Thermo Fisher Scientific, Inc., Waltham, MA, USA), and $5 \mathrm{~mL}$ penicillin/streptomycin (Gibco; Thermo Fisher Scientific, Inc., Waltham, MA, USA). All chemicals were purchased from Sigma-Aldrich (St. Louis, MO, USA) unless otherwise stated. The concentrations of empagliflozin (Boehringer Ingelheim, Ingelheim, Germany) used were based on the published report and preclinical studies performed at Boehringer Ingelheim $[15,16]$.

\subsection{Cell Viability Assay}

Crystal violet assay was performed to examine the effect of empagliflozin on cell viability. Cell viability was measured using previously described protocols [14]. Cells were seeded in 96-well plates $\left(5 \times 10^{3}\right.$ cells/well). After an overnight incubation, cells were treated with high $(30 \mathrm{mM})$ or normal $(5 \mathrm{mM})$ concentration of glucose for $24 \mathrm{~h}$. [15]. Cells were treated with $0.5 \%$ crystal violet reagent in phosphate-buffered saline (PBS) for $2 \mathrm{~h}$ at $37^{\circ} \mathrm{C}$. The cells were washed 10 times with PBS and air-dried, followed by incubation with $100 \mathrm{~mL}$ of dimethyl sulfoxide for $1-2 \mathrm{~h}$ for complete dissolution of the dye. The absorbance of the reaction product was measured at $570 \mathrm{~nm}$ using a microplate reader.

\subsection{Western Blot Analysis}

Cells were seeded at a density of $3 \times 10^{5}$ cells/well in a six-well plate. After an overnight incubation, cells were incubated in normal or high glucose medium. Western blot analysis was carried out as previously described [17]. In brief, cell pellets were homogenized in a buffer and centrifuged at $14,000 \times g$. Protein $(40 \mu \mathrm{g})$ from the supernatant of each sample was separated with sodium dodecyl sulphate polyacrylamide gel electrophoresis (SDS-PAGE) and transferred onto polyvinylidene difluoride membranes by electrophoresis. The membranes were blocked in TBST buffer for $1 \mathrm{~h}$ at room temperature. The blots were probed with primary anti-MFN1 (sc-100561, Santa Cruz Biotechnology, Inc), anti-MFN2 (sc-100560, Santa Cruz Biotechnology, Inc), anti-DRP1 (sc-101270, Santa Cruz Biotechnology, Inc), and anti-FIS1 (GTX111010, GeneTex, Inc) antibodies, followed by incubation with secondary 
antibodies at an appropriate dilution. The blots were visualized with the Western Lightning Plus-ECL (PerkinElmer, Waltham, MA, USA).

\subsection{Mitochondrial Fragmentation Index}

Mitochondrial morphology was assayed as previously described with some modifications [14,18]. Briefly, mitochondria were stained by the fluorescent dye Mitotracker red (Invitrogen, Life Technologies, Carlsbad, CA, USA) and observed under a fluorescence microscope (Olympus BX-UCDB-2; Olympus Corporation, Tokyo, Japan). Mitochondrial morphology was scored in a blinded and independent manner by two experienced technicians. Mitochondria with punctated or fragmented patterns were termed as the fission type. The fission rate (\%) was defined as the percentage of fission type mitochondrion among all counted mitochondria, i.e., fission events/total (fission + intermediate + fusion types) $\times 100$.

\subsection{Cellular Apoptotic Assay}

In the terminal deoxynucleotidyl transferase dUTP nick end labelling (TUNEL) assay, HK-2 cells incubated for $24 \mathrm{~h}$ in the presence or absence of empagliflozin were fixed in $4 \%$ paraformaldehyde and treated with a blocking solution (10\% FBS in PBS) for $30 \mathrm{~min}$ at $25^{\circ} \mathrm{C}$. Cells were permeabilized with $0.1 \%(v / v)$ Triton X-100 in PBS, followed by incubation with the TUNEL reaction mixture (Roche, Mannheim, Germany) for $1 \mathrm{~h}$ at $37^{\circ} \mathrm{C}$. After the treatment, the cells were observed under a fluorescence microscope (Olympus BX-51; Olympus Corporation, Tokyo, Japan).

\subsection{Measurement of Mitochondrial Membrane Potential (MMP)}

Rhodamine 123 (Invitrogen, Life Technologies, Carlsbad, CA, USA) was used to measure MMP, as previously described [19]. Briefly, $2 \times 10^{5}$ cells were plated in each well of a six-well plate and allowed to attach for 16-18 h. After treatment for $48 \mathrm{~h}$, the cells were harvested with trypsin, washed with PBS, and resuspended in $200 \mathrm{ng} / \mathrm{mL}$ of Rhodamine 123 . After incubation for $30 \mathrm{~min}$ at $37^{\circ} \mathrm{C}$, the cells were washed thrice and resuspended in $500 \mathrm{~mL}$ of PBS. Cytofluorimetric analysis was performed using a BD Biosciences FACScan system.

\subsection{Quantification of Cellular and Mitochondrial ROS}

Cellular ROS level was detected using $2^{\prime}, 7^{\prime}$-dichlorodihydrofluorescein diacetate (DCFH-DA) as previously described [20]. Briefly, $2 \times 10^{5}$ cells were incubated with appropriate concentrations of glucose and empagliflozin for $24 \mathrm{~h}$. The cells were washed twice with PBS and incubated with $10 \mu \mathrm{M}$ DCFH-DA (Molecular Probes, Life Technologies, Carlsbad, CA, USA) for $30 \mathrm{~min}$ at $37^{\circ} \mathrm{C}$. Cells were harvested with trypsin/ethylenediaminetetraacetic acid (EDTA), centrifuged at $200 \times g$ for $5 \mathrm{~min}$, and resuspended in $0.5 \mathrm{~mL}$ of PBS. Cellular ROS level was measured by flow cytometry using a fluorescence-activated cell scanner FACScan machine (BD Biosciences Inc., San Jose, CA, USA). The mean fluorescence intensity (MFI) for each sample was calculated using the CellQuest software. Mitochondrial ROS level was calculated using MitoSOX Red (Invitrogen, Life Technologies, Carlsbad, CA, USA). Briefly, $2 \times 10^{5}$ cells were plated in each well of six-well plates and allowed to attach for 16-18 h. After being treated with drugs for $24 \mathrm{~h}$, the cells were harvested by trypsin treatment, washed in PBS, and resuspended in $5 \mu \mathrm{M}$ MitoSOX red. After incubation for $30 \mathrm{~min}$ at $37^{\circ} \mathrm{C}$, the cells were washed thrice and resuspended in $500 \mu \mathrm{L}$ of PBS. Cytofluorimetric analysis data were similar to those of cellular ROS quantitation.

\subsection{Measurement of ATP Generation}

ATP content was colorimetrically determined with the ATP Colorimetric/Fluorometric Assay kit (BioVision, Milpitas, CA, USA) following the manufacturer's instructions. Briefly, $1 \times 10^{6}$ cells were maintained in a T75 flask overnight. Treatment groups included $5 \mathrm{mM}$ glucose, $30 \mathrm{mM}$ glucose, $30 \mathrm{mM}$ 
glucose $+100 \mathrm{nM}$ empagliflozin, and $30 \mathrm{mM}$ glucose $+500 \mathrm{nM}$ empagliflozin groups. After overnight treatment, the cells were harvested, collected by centrifugation at $200 \times g$ for $3 \mathrm{~min}$, washed with PBS, and lysed in $100 \mu \mathrm{L}$ ATP assay buffer. The samples or standards (50 $\mu \mathrm{L}$ per well) and the reaction

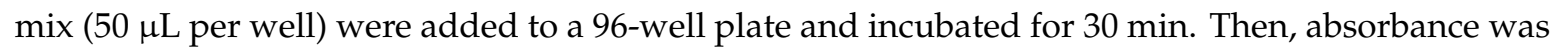
measured at $570 \mathrm{~nm}$ using an ELISA reader Quant (BioTek Instruments Inc., Winooski, VT, USA)

\subsection{Statistical Analysis}

All experiments were repeated at least three times. The quantitative data are presented as mean \pm standard error of the mean (SEM). Statistical analyses were performed using IBM SPSS Statistics for Windows, Version 19.0. (IBM Corp., Armonk, New York, NY, USA). Comparisons between multiple groups were carried out using one-way analysis of variance (ANOVA). Pair-wise comparisons were performed using the $t$-test. A value of $P<0.05$ was considered significant.

\section{Results}

\subsection{Empagliflozin Exerts Insignificant Effects on Cell Viability}

The viability of HK-2 cells treated with the indicated concentrations of glucose and empagliflozin is shown in Figure 1. We tested empagliflozin at concentrations of 100, 500, 1000, and $2000 \mathrm{nM}$ under normal $(5 \mathrm{mM})$ and high glucose $(30 \mathrm{mM})$ conditions. Treatment with the fixed concentration of acetonitrile, which was used as the solvent for empagliflozin, was considered as vehicle control. Empagliflozin did not affect cellular viability. In comparison with the normal glucose condition, high glucose condition caused a 12\% reduction in cell viability (Figure 1). In addition to demonstrating that empagliflozin exerts insignificant effects on cell viability, we performed validation experiments including glucose uptake and RNA interference to silence SGLT2. These validation experiments were to confirm the primary effects of empagliflozin on HK-2 cells and to support that our pharmacology experiment results were specifically due to SGLT2 inhibition (Supplementary Material).

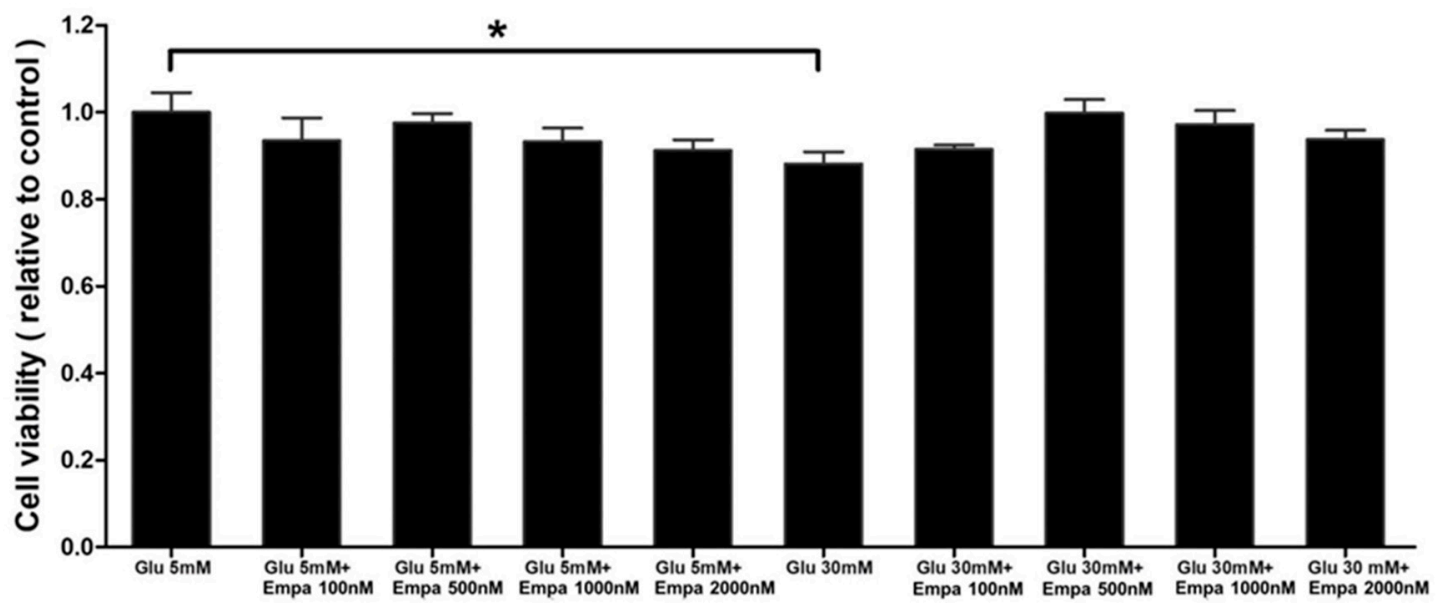

Figure 1. Empagliflozin has no negative effects on the viability of HK-2 cells. Cell viability was measured with crystal violet assay and the absorbance was analyzed at $570 \mathrm{~nm}$ using a microplate reader. Data were obtained from three independent experiments and are expressed as mean \pm SEM. Only high glucose $(30 \mathrm{mM})$ treatment led to a decrease in cell viability. ${ }^{*} P<0.05$ versus the normal glucose (5 mM) treatment group. Glu, glucose; Empa, empagliflozin.

\subsection{Empagliflozin Rescues High Glucose-Induced Mitochondrial Fragmentation in Human PTCs}

Earlier, we have demonstrated the effect of high glucose on mitochondrial fragmentation in human PTCs [14]. We, therefore, investigated whether empagliflozin rescues this effect. Figure 2A-D shows the morphological features of mitochondria from the four treatment groups. Magnified images of 
indexed mitochondria in each treatment group are shown in Figure 2E-H. We found that HK-2 cells were susceptible to high glucose-induced mitochondrial fragmentation, and empagliflozin could rescue mitochondrial fragmentation. To quantitatively analyze the process of mitochondrial fragmentation, we calculated the mitochondrial fission rate in each treatment group. High glucose $(30 \mathrm{mM})$ condition led to a four-times higher fission rate in HK-2 cells than the normal glucose condition. In the presence of high glucose, empagliflozin at either 100 or $500 \mathrm{nM}$ concentration could reduce the mitochondrial fission rate to a level comparable to that reported under normal glucose condition (Figure 2I).
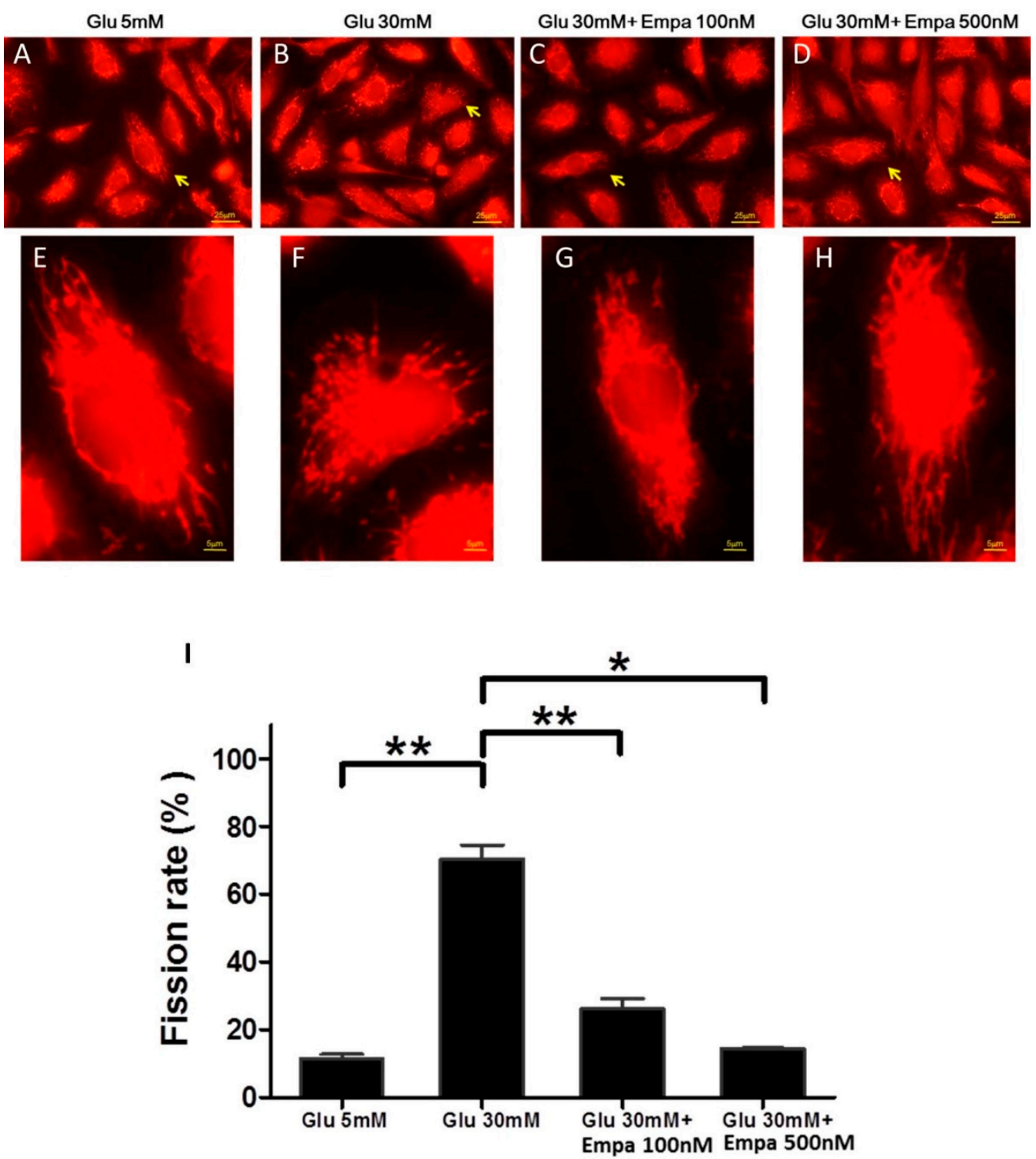

Figure 2. Empagliflozin rescues high glucose-induced mitochondrial fragmentation in human PTCs. HK-2 cells were cultured in $5 \mathrm{mM}$ glucose (A), $30 \mathrm{mM}$ glucose (B), $30 \mathrm{mM}$ glucose with $100 \mathrm{nM}$ empagliflozin (C), and $30 \mathrm{mM}$ glucose with $500 \mathrm{nM}$ empagliflozin (D). Mitochondria were stained with Mitotracker red. Magnified images of indexed mitochondria (arrow) in each treatment group are shown in (E-H). (I) The mitochondrial fission rate was higher in high glucose condition. Empagliflozin rescued this effect. Data were obtained from three independent experiments and are expressed as mean \pm SEM. ${ }^{*} P<0.05,{ }^{* *} P<0.001$. 


\subsection{Impacts of High Glucose and Empagliflozin on the Expression of Mitochondrial Fusion/Fission Proteins}

Western blot analysis (Figure 3A) revealed the high glucose-induced downregulation in the expression of fusion proteins (i.e., MFN1 and MFN2, Figure 3B,C). Empagliflozin could reverse the high glucose-induced downregulation of MFN1 and MFN2 expression at 500 and $100 \mathrm{nM}$ concentration, respectively (Figure 3B,C). High glucose failed to cause any changes in the expression of fission proteins (i.e., DRP1 and FIS1, Figure 3D,E). In the presence of high glucose, empagliflozin could downregulate the expression of DRP1 at $100 \mathrm{nM}$ concentration. Similar effects were observed on FIS1 expression in the presence of $500 \mathrm{nM}$ empagliflozin (Figure 3D,E). As mitochondrial morphology is controlled by the balance between fusion/fission proteins, the results shown in Figure 3 support the observation illustrated in Figure 2.
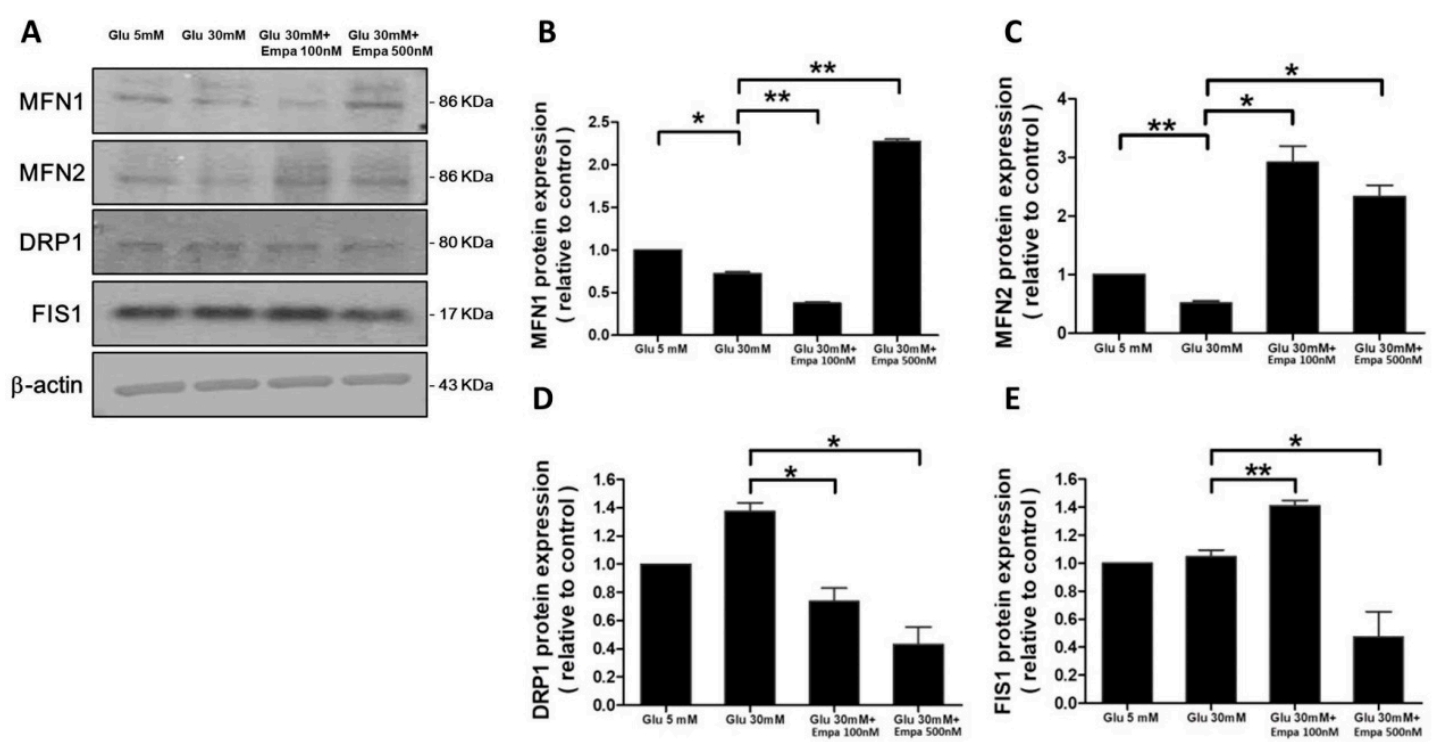

Figure 3. Impact of empagliflozin on high glucose-induced alterations in the expression levels of mitochondrial fusion/fission proteins. (A-E) The expression of MFN1, MFN2, DRP1, and FIS1 was analyzed with western blotting and normalized to the level of $\beta$-actin. Data are expressed as mean \pm SEM. ${ }^{*} P<0.05,{ }^{* *} P<0.001$.

\subsection{Empagliflozin Reduces the High Glucose-Induced Cellular Apoptosis of HK-2 Cells}

Compared to the normal glucose condition (Figure 4A), high glucose condition induced a marked increase in the level of cellular apoptosis (Figure 4B). Empagliflozin at 100 and $500 \mathrm{nM}$ concentrations offered effective protection against high glucose-induced apoptosis (Figure 4C and 4D). Quantitative analysis of TUNEL assay result showed that high glucose markedly induced apoptosis and that empagliflozin could ameliorate this effect (Figure 4E). 

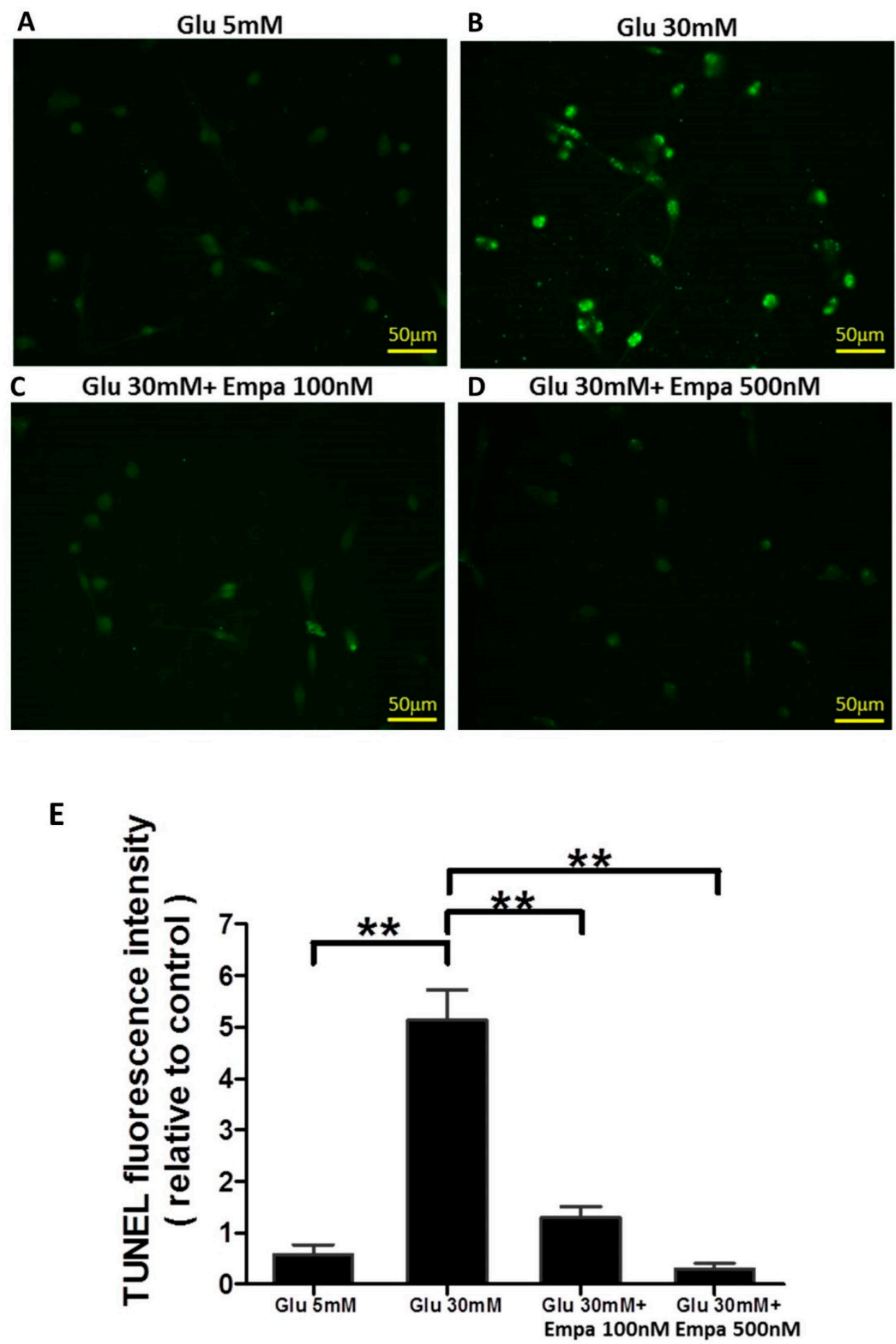

Figure 4. Empagliflozin reduces the high glucose-induced apoptosis of HK-2 cells. (A-D) Fluorescence images show positive TUNEL staining in the four treatment groups. (E) Quantitative analysis of TUNEL assay shows that high glucose markedly induces apoptosis and empagliflozin ameliorates this effect. Data are expressed as mean \pm SEM. ${ }^{* *} P<0.001$.

\subsection{Empagliflozin Improves Mitochondrial Function in High Glucose-Treated HK-2 Cells}

We examined mitochondrial function of HK-2 cells by analyzing cellular (Figure 5A) and mitochondrial ROS production (Figure 5B), MMP (Figure 5C), and ATP generation (Figure 5D). High glucose increased both cellular and mitochondrial ROS levels. Empagliflozin at $500 \mathrm{nM}$ could reduce the cellular ROS level, although $100 \mathrm{nM}$ empagliflozin was not sufficiently effective (Figure 5A). 
Empagliflozin at 100 and $500 \mathrm{nM}$ concentrations could maintain the mitochondrial ROS production level (Figure 5B). High glucose caused a decrease in MMP and this effect was ameliorated by empagliflozin at $500 \mathrm{nM}$ concentration (Figure 5C). Empagliflozin at a concentration of either 100 or $500 \mathrm{nM}$ was able to increase the level of ATP in HK-2 cells (Figure 5D). Taken together, the results of mitochondrial functional assays show that empagliflozin at $500 \mathrm{nM}$ concentration produced healthier mitochondria to combat the stress induced by high glucose.

A

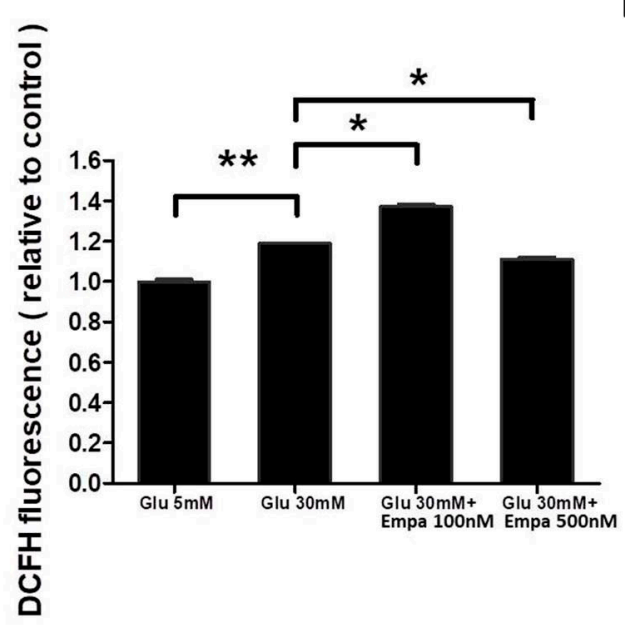

C

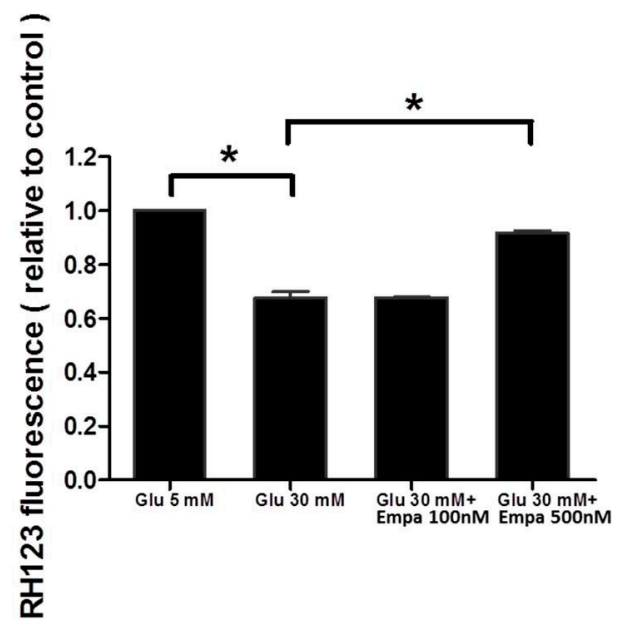

B

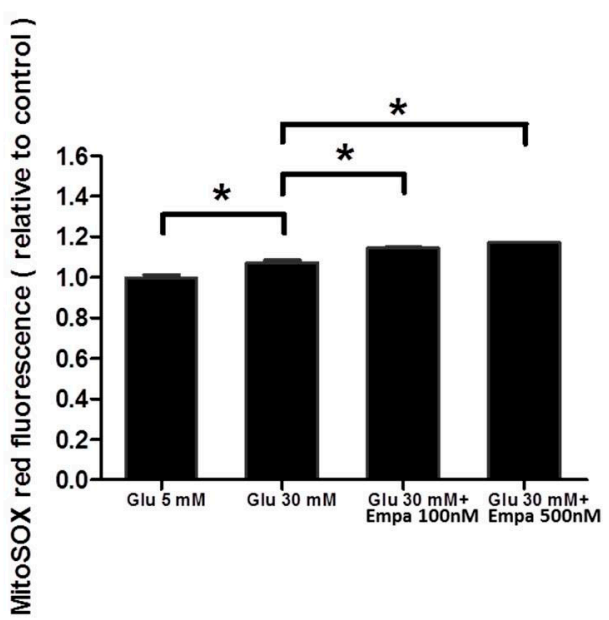

D

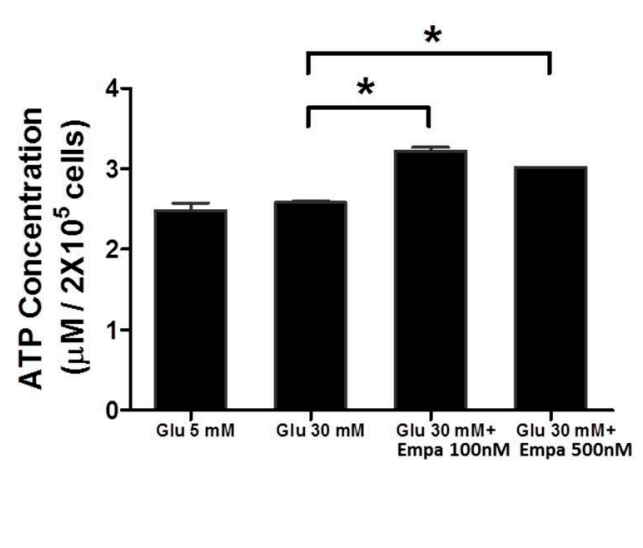

Figure 5. Empagliflozin improves the mitochondrial function of high glucose-treated HK-2 cells. (A) Cellular ROS production, (B) mitochondrial ROS production, (C) MMP, and (D) ATP generation in the four treatment groups. Data were obtained from three independent experiments and are expressed as mean \pm SEM. ${ }^{*} P<0.05,{ }^{* *} P<0.001$.

\section{Discussion}

Complimentary to the traditional 'glomerulocentric' perspective of diabetic nephropathy, the understanding of the pathophysiological perturbations in the tubules is essential for the successful management of DKD. In the diabetic milieu, increased energy demands and a decreased perfusion result in tubular hypoxia. This phenomenon may drive the development of tubular atrophy and interstitial fibrosis in a vicious cycle that worsens the clinical course of DKD [21]. At the subcellular level, the mitochondrion of diabetic proximal tubules is the key organelle that modulates its propensity to hypoxic injury and oxidative stress. Studies on subcellular levels often unravel the underlying mechanisms that clinical trials may not reach. Here, we investigated the subcellular mechanisms underlying the protective effect of empagliflozin from high glucose-mediated injuries on PTCs. As oxidative stress 
plays pathologic roles in diabetic kidneys [22-24] and empagliflozin may reduce this effect $[25,26]$, we analyzed the direct effects of empagliflozin on the mitochondria of PTCs.

In PTCs, high glucose treatment induces mitochondrial fragmentation and cellular apoptosis [14,27]. Our results showed that empagliflozin protected PTCs from high glucose-induced injuries through the modulation of the expression of mitochondrial fusion and fission proteins. We demonstrate that empagliflozin could rescue high glucose-induced mitochondrial fragmentation. At the protein level, high glucose condition significantly downregulated the expression levels of both mitochondrial fusion proteins, while empagliflozin treatment rescued this effect by enhancing the expression levels of both mitochondrial fusion proteins. Although not significant, high glucose seemed to cause an upregulation in the expression levels of mitochondrial fission proteins, which were significantly downregulated by empagliflozin. In line with the proposed protective mechanism in a different cellular aspect [28], empagliflozin might make mitochondria more resistant to high glucose-driven fragmentation by upregulating the expression of fusion proteins and downregulating the levels of fission proteins. This protective mechanism may explain why empagliflozin protected PTCs from high glucose-induced mitochondrial fragmentation and cellular apoptosis.

In addition to shaping the mitochondrial morphology, empagliflozin could also improve mitochondrial functions. Mitochondrial dysfunction has been reported in experimental diabetic animal and cellular models [29-31]. These disturbances in mitochondrial functions may result in the excessive accumulation of cellular ROS [30,32], ATP depletion [33], and MMP reduction [30]. Our results confirm these observations in response to high glucose-induced mitochondrial dysfunctions in PTCs and demonstrate that empagliflozin could ameliorate these effects. In DKD, ROS is produced from various organelles within the cell, including the mitochondrion, endoplasmic reticulum, and the enzyme systems such as Nox. ROS underproduction may be indicative of the mitochondrial dysfunction. Steady-state levels of ROS are involved in normal cellular signaling, whereas excessive ROS production leads to cellular dysfunction [34]. Our results demonstrate that empagliflozin reduced the cellular ROS level while maintaining the mitochondrial ROS production.

The mitochondrion has been recognized as a crucial target for DKD treatment [35,36]. Several mitochondrion-targeted agents, including MitoQ [37], Ebselen [38], Bendavia [39], and MitoTEMPO [40], have been reported. Although the molecular pathways underlying the empagliflozin-mediated increase in mitochondrial fitness are not completely understood, our results indicated that empagliflozin could serve as a mitochondrion-targeting agent. Proximal tubules, the direct site of action of empagliflozin, emerge as a novel therapeutic target for DKD [21]. This study revealed a novel mechanism explaining the renal benefits of empagliflozin, as demonstrated in the EMPA-REG OUTCOME trial, and this warrants future studies to identify its role in diabetic tubulopathy.

\section{Conclusions}

In conclusion, we demonstrated that empagliflozin treatment improves mitochondrial performance in HK-2 cells, as reflected from their morphologies and functions, to overcome high glucose-induced injuries.

Supplementary Materials: The following are available online at http://www.mdpi.com/2073-4409/8/9/1085/s1, Figure S1: Empagliflozin reduces intracellular glucose. Intracellular glucose levels were measured by glucose uptake assay; Figure S2: SGLT2 knocked down by siSGLT2. In HK-2 cells, SGLT2 was effectively knocked down by siSGLT2 at the concentration of 20 and $30 \mathrm{nM}$. NC, negative control; SC, scrambled siRNA; Figure S3: Silencing of SGLT2 reduces the high glucose-induced apoptosis of HK-2 cells; Figure S4: Empagliflozin and siSGLT2 reduce high glucose-induced cellular ROS production in HK-2 cells.

Author Contributions: Conceptualization, W.-C.L., Y.-Y.C., H.-Y.N., P.-W.W., C.-W.L., T.-K.L., and J.-B.C.; Methodology, W.-C.L., H.-Y.N., and C.-H.C.; Software, C.-H.C.; Validation, W.-C.L., Y.-Y.C., and H.-Y.N.; Formal analysis, W.-C.L. and H.-Y.N.; Investigation, W.-C.L.; Resources, W.-C.L.; Data curation, W.-C.L. and C.-H.C.; Writing-original draft preparation, W.-C.L.; Writing-review and editing, W.-C.L.; Visualization, W.-C.L.; Supervision, J.-B.C.; Project administration, W.-C.L. and J.-B.C.; Funding acquisition, W.-C.L.

Funding: This research was funded by Kaohsiung Chang Gung Memorial Hospital (grant number CMRPG8F1451). 
Conflicts of Interest: The authors declare no conflict of interest. The funders had no role in the design of the study; in the collection, analyses, or interpretation of data; in the writing of the manuscript, or in the decision to publish the results.

\section{References}

1. Alicic, R.Z.; Rooney, M.T.; Tuttle, K.R. Diabetic kidney disease: Challenges, progress, and possibilities. Clin. J. Am. Soc. Nephrol. 2017, 12, 2032-2045. [CrossRef] [PubMed]

2. de Boer, I.H.; Rue, T.C.; Hall, Y.N.; Heagerty, P.J.; Weiss, N.S.; Himmelfarb, J. Temporal trends in the prevalence of diabetic kidney disease in the united states. JAMA 2011, 305, 2532-2539. [CrossRef] [PubMed]

3. Lee, W.C.; Lee, Y.T.; Li, L.C.; Ng, H.Y.; Kuo, W.H.; Lin, P.T.; Liao, Y.C.; Chiou, T.T.; Lee, C.T. The number of comorbidities predicts renal outcomes in patients with stage $3(-) 5$ chronic kidney disease. J. Clin. Med. 2018, 7, 493. [CrossRef] [PubMed]

4. Wanner, C.; Inzucchi, S.E.; Lachin, J.M.; Fitchett, D.; von Eynatten, M.; Mattheus, M.; Johansen, O.E.; Woerle, H.J.; Broedl, U.C.; Zinman, B.; et al. Empagliflozin and progression of kidney disease in type 2 diabetes. N. Engl. J. Med. 2016, 375, 323-334. [CrossRef] [PubMed]

5. Nagata, T.; Fukuzawa, T.; Takeda, M.; Fukazawa, M.; Mori, T.; Nihei, T.; Honda, K.; Suzuki, Y.; Kawabe, Y. Tofogliflozin, a novel sodium-glucose co-transporter 2 inhibitor, improves renal and pancreatic function in db/db mice. Br. J. Pharmacol. 2013, 170, 519-531. [CrossRef]

6. Vallon, V.; Gerasimova, M.; Rose, M.A.; Masuda, T.; Satriano, J.; Mayoux, E.; Koepsell, H.; Thomson, S.C.; Rieg, T. Sglt2 inhibitor empagliflozin reduces renal growth and albuminuria in proportion to hyperglycemia and prevents glomerular hyperfiltration in diabetic akita mice. Am. J. Physiol. Renal. Physiol. 2014, 306, F194-F204. [CrossRef]

7. Gembardt, F.; Bartaun, C.; Jarzebska, N.; Mayoux, E.; Todorov, V.T.; Hohenstein, B.; Hugo, C. The sglt2 inhibitor empagliflozin ameliorates early features of diabetic nephropathy in btbr ob/ob type 2 diabetic mice with and without hypertension. Am. J. Physiol. Renal. Physiol. 2014, 307, F317-F325. [CrossRef] [PubMed]

8. Heerspink, H.J.; Perkins, B.A.; Fitchett, D.H.; Husain, M.; Cherney, D.Z. Sodium glucose cotransporter 2 inhibitors in the treatment of diabetes mellitus: Cardiovascular and kidney effects, potential mechanisms, and clinical applications. Circulation 2016, 134, 752-772. [CrossRef]

9. Hakim, F.A.; Pflueger, A. Role of oxidative stress in diabetic kidney disease. Med. Sci. Monit. 2010, 16, RA37-RA48.

10. Weng, S.W.; Lin, T.K.; Liou, C.W.; Chen, S.D.; Wei, Y.H.; Lee, H.C.; Chen, I.Y.; Hsieh, C.J.; Wang, P.W. Peripheral blood mitochondrial DNA content and dysregulation of glucose metabolism. Diabetes Res. Clin. Pract. 2009, 83, 94-99. [CrossRef]

11. Takebayashi, S.; Kaneda, K. Mitochondrial derangement: Possible initiator of microalbuminuria in niddm. J. Diabet.Complications 1991, 5, 104-106. [CrossRef]

12. Chen, H.; Detmer, S.A.; Ewald, A.J.; Griffin, E.E.; Fraser, S.E.; Chan, D.C. Mitofusins mfn1 and mfn2 coordinately regulate mitochondrial fusion and are essential for embryonic development. J. Cell Biol. 2003, 160, 189-200. [CrossRef]

13. Santel, A.; Fuller, M.T. Control of mitochondrial morphology by a human mitofusin. J. Cell Sci. 2001, 114, 867-874.

14. Lee, W.C.; Chiu, C.H.; Chen, J.B.; Chen, C.H.; Chang, H.W. Mitochondrial fission increases apoptosis and decreases autophagy in renal proximal tubular epithelial cells treated with high glucose. DNA Cell Biol. 2016, 35, 657-665. [CrossRef]

15. Panchapakesan, U.; Pegg, K.; Gross, S.; Komala, M.G.; Mudaliar, H.; Forbes, J.; Pollock, C.; Mather, A. Effects of sglt2 inhibition in human kidney proximal tubular cells-Renoprotection in diabetic nephropathy? PLoS ONE 2013, 8, e54442. [CrossRef]

16. Grempler, R.; Thomas, L.; Eckhardt, M.; Himmelsbach, F.; Sauer, A.; Sharp, D.E.; Bakker, R.A.; Mark, M.; Klein, T.; Eickelmann, P. Empagliflozin, a novel selective sodium glucose cotransporter-2 (sglt-2) inhibitor: Characterisation and comparison with other sglt-2 inhibitors. Diabetes Obes. Metab. 2012, 14, 83-90. [CrossRef] 
17. Tain, Y.L.; Lee, W.C.; Hsu, C.N.; Lee, W.C.; Huang, L.T.; Lee, C.T.; Lin, C.Y. Asymmetric dimethylarginine is associated with developmental programming of adult kidney disease and hypertension in offspring of streptozotocin-treated mothers. PLOS ONE 2013, 8, e55420. [CrossRef]

18. Brooks, C.; Wei, Q.; Feng, L.; Dong, G.; Tao, Y.; Mei, L.; Xie, Z.J.; Dong, Z. Bak regulates mitochondrial morphology and pathology during apoptosis by interacting with mitofusins. Proc. Natl. Acad. Sci. USA 2007, 104, 11649-11654. [CrossRef]

19. Tiao, M.M.; Liou, C.W.; Huang, L.T.; Wang, P.W.; Lin, T.K.; Chen, J.B.; Chou, Y.M.; Huang, Y.H.; Lin, H.Y.; Chen, C.L.; et al. Associations of mitochondrial haplogroups b4 and e with biliary atresia and differential susceptibility to hydrophobic bile acid. PLoS Genet 2013, 9, e1003696. [CrossRef]

20. Lee, W.C.; Li, L.C.; Chen, J.B.; Chang, H.W. Indoxyl sulfate-induced oxidative stress, mitochondrial dysfunction, and impaired biogenesis are partly protected by vitamin c and n-acetylcysteine. Sci. World J. 2015, 2015, 620826. [CrossRef]

21. Gilbert, R.E. Proximal tubulopathy: Prime mover and key therapeutic target in diabetic kidney disease. Diabetes 2017, 66, 791-800. [CrossRef]

22. Aghadavod, E.; Khodadadi, S.; Baradaran, A.; Nasri, P.; Bahmani, M.; Rafieian-Kopaei, M. Role of oxidative stress and inflammatory factors in diabetic kidney disease. Iran J. Kidney Dis. 2016, 10, 337-343.

23. Arora, M.K.; Singh, U.K. Oxidative stress: Meeting multiple targets in pathogenesis of diabetic nephropathy. Curr. Drug Targets 2014, 15, 531-538. [CrossRef]

24. Singh, D.K.; Winocour, P.; Farrington, K. Oxidative stress in early diabetic nephropathy: Fueling the fire. Nat. Rev. Endocrinol. 2011, 7, 176-184. [CrossRef]

25. Andreadou, I.; Efentakis, P.; Balafas, E.; Togliatto, G.; Davos, C.H.; Varela, A.; Dimitriou, C.A.; Nikolaou, P.E.; Maratou, E.; Lambadiari, V.; et al. Empagliflozin limits myocardial infarction in vivo and cell death in vitro: Role of stat3, mitochondria, and redox aspects. Front. Physiol. 2017, 8, 1077. [CrossRef]

26. Steven, S.; Oelze, M.; Hanf, A.; Kroller-Schon, S.; Kashani, F.; Roohani, S.; Welschof, P.; Kopp, M.; Godtel-Armbrust, U.; Xia, N.; et al. The sglt2 inhibitor empagliflozin improves the primary diabetic complications in zdf rats. Redox Biol. 2017, 13, 370-385. [CrossRef]

27. Zhan, M.; Usman, I.M.; Sun, L.; Kanwar, Y.S. Disruption of renal tubular mitochondrial quality control by myo-inositol oxygenase in diabetic kidney disease. J. Am. Soc. Nephrol. 2015, 26, 1304-1321. [CrossRef]

28. van der Bliek, A.M. Fussy mitochondria fuse in response to stress. EMBO J. 2009, 28, 1533-1534. [CrossRef]

29. Coughlan, M.T.; Thorburn, D.R.; Penfold, S.A.; Laskowski, A.; Harcourt, B.E.; Sourris, K.C.; Tan, A.L.; Fukami, K.; Thallas-Bonke, V.; Nawroth, P.P.; et al. Rage-induced cytosolic ros promote mitochondrial superoxide generation in diabetes. J. Am. Soc. Nephrol. 2009, 20, 742-752. [CrossRef]

30. Gao, C.L.; Zhu, C.; Zhao, Y.P.; Chen, X.H.; Ji, C.B.; Zhang, C.M.; Zhu, J.G.; Xia, Z.K.; Tong, M.L.; Guo, X.R. Mitochondrial dysfunction is induced by high levels of glucose and free fatty acids in 3t3-11 adipocytes. Mol. Cell. Endocrinol. 2010, 320, 25-33. [CrossRef]

31. Rosca, M.G.; Mustata, T.G.; Kinter, M.T.; Ozdemir, A.M.; Kern, T.S.; Szweda, L.I.; Brownlee, M.; Monnier, V.M.; Weiss, M.F. Glycation of mitochondrial proteins from diabetic rat kidney is associated with excess superoxide formation. Am. J. Physiol. Renal. Physiol. 2005, 289, F420-F430. [CrossRef]

32. Brownlee, M. The pathobiology of diabetic complications: A unifying mechanism. Diabetes 2005, 54, 1615-1625. [CrossRef]

33. Tan, A.L.; Sourris, K.C.; Harcourt, B.E.; Thallas-Bonke, V.; Penfold, S.; Andrikopoulos, S.; Thomas, M.C.; O’Brien, R.C.; Bierhaus, A.; Cooper, M.E.; et al. Disparate effects on renal and oxidative parameters following rage deletion, age accumulation inhibition, or dietary age control in experimental diabetic nephropathy. Am. J. Physiol. Renal Physiol. 2010, 298, F763-F770. [CrossRef]

34. Coughlan, M.T.; Sharma, K. Challenging the dogma of mitochondrial reactive oxygen species overproduction in diabetic kidney disease. Kidney Int. 2016, 90, 272-279. [CrossRef]

35. Galvan, D.L.; Green, N.H.; Danesh, F.R. The hallmarks of mitochondrial dysfunction in chronic kidney disease. Kidney Int. 2017, 92, 1051-1057. [CrossRef]

36. Sharma, K. Mitochondrial dysfunction in the diabetic kidney. Adv. Exp. Med. Biol. 2017, 982, 553-562.

37. Xiao, L.; Xu, X.; Zhang, F.; Wang, M.; Xu, Y.; Tang, D.; Wang, J.; Qin, Y.; Liu, Y.; Tang, C.; et al. The mitochondria-targeted antioxidant mitoq ameliorated tubular injury mediated by mitophagy in diabetic kidney disease via nrf2/pink1. Redox Biol. 2017, 11, 297-311. [CrossRef] 
38. Filipovska, A.; Kelso, G.F.; Brown, S.E.; Beer, S.M.; Smith, R.A.; Murphy, M.P. Synthesis and characterization of a triphenylphosphonium-conjugated peroxidase mimetic. Insights into the interaction of ebselen with mitochondria. J. Biol. Chem. 2005, 280, 24113-24126. [CrossRef]

39. Szeto, H.H.; Liu, S.; Soong, Y.; Wu, D.; Darrah, S.F.; Cheng, F.Y.; Zhao, Z.; Ganger, M.; Tow, C.Y.; Seshan, S.V. Mitochondria-targeted peptide accelerates atp recovery and reduces ischemic kidney injury. J. Am. Soc. Nephrol. 2011, 22, 1041-1052. [CrossRef]

40. Liang, H.L.; Sedlic, F.; Bosnjak, Z.; Nilakantan, V. Sod1 and mitotempo partially prevent mitochondrial permeability transition pore opening, necrosis, and mitochondrial apoptosis after atp depletion recovery. Free Radic. Biol. Med. 2010, 49, 1550-1560. [CrossRef]

(C) 2019 by the authors. Licensee MDPI, Basel, Switzerland. This article is an open access article distributed under the terms and conditions of the Creative Commons Attribution (CC BY) license (http://creativecommons.org/licenses/by/4.0/). 